\title{
COVID-19 Outbreak: Infection Control and Management Protocol for Vascular and Interventional Radiology Departments-Consensus Document
}

\author{
Miguel A. De Gregorio ${ }^{1} \cdot$ Jose A. Guirola $^{1}($ D $)$ Mariano Magallanes ${ }^{2}$ • \\ Julio Palmero ${ }^{3} \cdot$ Juan M. Pulido $^{4} \cdot$ Javier Blazquez $^{5} \cdot$ Jorge Cobos $^{5}$. \\ Jose M. Abadal ${ }^{6} \cdot$ Santiago Mendez $^{7} \cdot$ Mercedes Perez-Lafuente $^{8} \cdot$ Maria C. Piquero \\ Micheto $^{1}$ - Abel Gregorio ${ }^{9}$ Elena Lonjedo ${ }^{10}$. Teresa Moreno ${ }^{11} \cdot$ Jose R. Pulpeiro $^{12}$. $^{2}$ \\ Jaume Sampere $^{13} \cdot$ Enrique Esteban $^{14} \cdot$ Jose J. Muñoz $^{15} \cdot$ Jordi Bosch $^{1}$ • \\ Enrique Alvarez-Arranz ${ }^{1}$. Jimena Gonzalez ${ }^{16}$ • Arantxa Gelabert ${ }^{17}$. \\ Jose Urbano ${ }^{5}$ C COVID-19-IRSpain
}

Received: 31 March 2020/Accepted: 13 April 2020/Published online: 20 May 2020

(C) Springer Science+Business Media, LLC, part of Springer Nature and the Cardiovascular and Interventional Radiological Society of Europe (CIRSE) 2020

\begin{abstract}
COVID-19 (SARS-CoV-2 virus) pandemic was recently declared by the WHO as a global health emergency. A group of interventional radiology senior experts developed a consensus document for infection control and management of patients with COVID-19 in interventional radiology (IR) departments. This consensus statement has
\end{abstract}

Jose A. Guirola

joseandresguirola@gmail.com

Miguel A. De Gregorio

mgregori@unizar.es

Mariano Magallanes

mmagallanes@povisa.es

Julio Palmero

palmero_jul@gva.es

Juan M. Pulido

juanpulidoduque@yahoo.es

Javier Blazquez

javier.blazquez@salud.madrid.org

Jorge Cobos

jorge.cobos.alonso@gmail.com

Jose M. Abadal

jmabadal@yahoo.es

Santiago Mendez

smendez.sma@gmail.com

Mercedes Perez-Lafuente

merchebrea@yahoo.es

Maria C. Piquero Micheto

mcpiqueromicheto@gmail.com

Abel Gregorio

abelgregorio@gmail.com been brought together at short notice with the help of different protocols developed by governmental entities and scientific societies to be adapted to the current reality and needs of IR Departments. Recommendations are the specific strategies to follow in IR departments, preventive measures and regulations, step by step for donning and

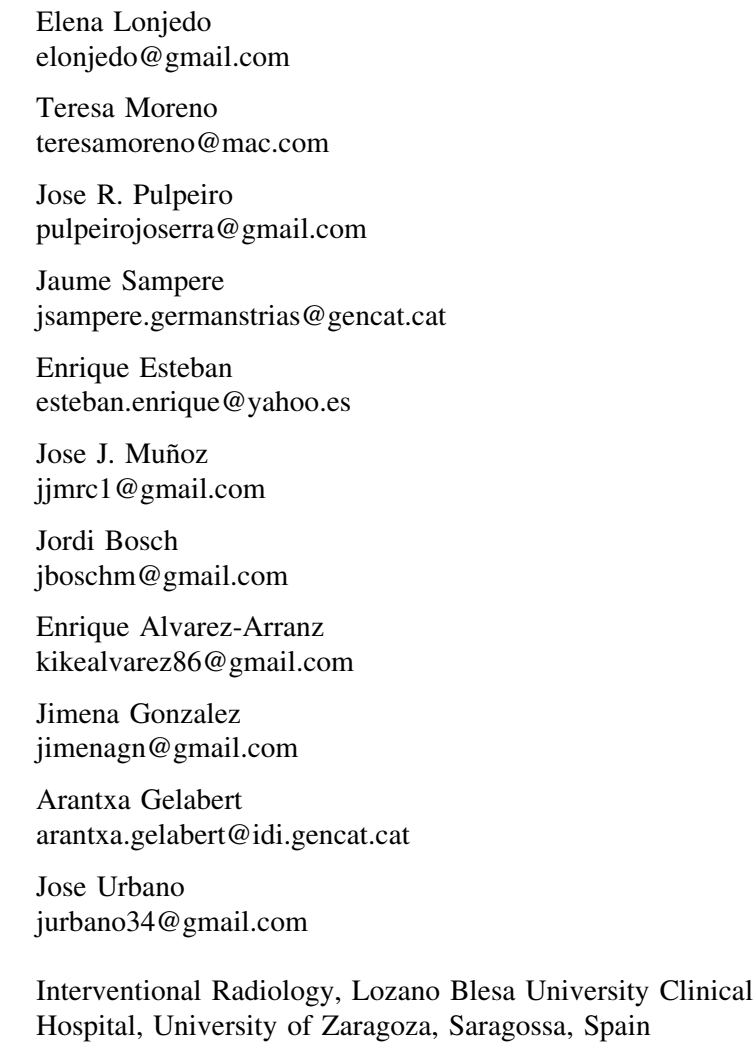


doffing personal protective equipment, specific IR procedures which can not be delayed, and aerosol-generating procedures in IR with COVID-19 patients. It is advisable with this document to be adapted to local workplace policies.

Keywords COVID-19 - Coronavirus infection control - Interventional suite infection control . Prevention of infection control · Health workers . Interventional radiologist safety $\cdot$ Interventional radiology staff safety COVID-19 patient management

\section{Introduction}

Infection with COVID-19 (SARS-CoV-2 virus) has recently been declared by the World Health Organization (WHO) as a global health emergency [1]. In late December of 2019, a heavily populated city of 11 million people in the Hubei province of China was the epicenter of the known pandemic due to severe acute respiratory syndrome (SARS) called SARS-CoV-2 or coronavirus disease of 2019 (COVID-19) [2].

On March 15, 2020, the Government of Spain declared the State of Alarm [3] due to the level of spread of the disease, with more than 13,897 deceased patients, 140,511 infected patients and 7,069 serious, critical patients (data of April 7, 2020) [4]. In the face of this alarming situation created by the outbreak of COVID-19, the vast majority of the interventional radiology (IR) interventional suites have altered their usual work following the instructions of the national and local health authorities. Among many Interventional Radiologists (IRs), there is a special concern

2 Interventional Radiology, Povisa Hospital, Vigo, Spain

3 Interventional Radiology, University Clinical Hospital of Valencia, Valencia, Spain

4 Interventional Radiology, Dr. Negrín University Hospital of Gran Canaria, Las Palmas de Gran Canaria, Spain

5 Interventional Radiology, Ramón y Cajal Hospital, Madrid, Spain

6 Interventional Radiology, Severo Ochoa Hospital, Leganés, Madrid, Spain

7 Interventional Radiology, Puerta de Hierro Hospital, Majadahonda, Madrid, Spain

8 Interventional Radiology, Vall d'Hebron Hospital, Barcelona, Spain

9 Interventional Radiology, Denia-Marina Salud Hospital, Denia, Alicante, Spain regarding the protocols of management in diagnosed COVID-19-patients, and especially in those cases that are unconfirmed or are suspected [5]. It is important to emphasize that the infection of COVID-19 in health workers is as high as $15 \%$ (more than 12,298) in Spain [6]. The government for the first time recognizes the shortage of personal protective equipment (PPE) and FFP2/FFP3 masks.

The Spanish Society of Vascular and Interventional Radiology (SERVEI) is aware that the IR plays an important role in the clinical and therapeutic management of many types of patients and wants to remain committed to these patients, attending to all the medical or surgical emergencies whether or not they are infected with COVID19.

The IR units, in general, are made up of a small number of IRs, residents, fellows, other specialist physicians, IRspecialized nurses, healthcare assistants, hospital orderlies or porters, radiology technicians, cleaning staff and medical physicist who carry out daily work in confined spaces with a high risk of infection by the overcrowding of healthcare personnel. These circumstances present highrisk inpatient or outpatient care, especially in the face of a possible forced quarantine of any IR staff who has been infected. There are many general rules and regulations that are constantly being disclosed in the hospitals; however, IRs and the interventional suites have some peculiarities that are not always considered. The three most important difficulties are the following: the scarce number of IRs to work in normal shifts (an average in Spain of 3.41 IRs per hospital), but also their availability during $24 \mathrm{~h}$ and 7 days a week on-call coverage [7, 8]; the lack of PPE and FFP2/ FFP3 masks; and interventional suites with multiple devices and equipment that have difficulty in sterilizing or preventing fomite transmission.

10 Interventional Radiology, Dr Peset Hospital, Valencia, Spain

11 Interventional Radiology, Juan Ramón Jiménez Hospital, Huelva, Spain

12 Interventional Radiology, Lucus Augusti Hospital, Lugo, Spain

13 Interventional Radiology, Germans Trias y Pujol Hospital, Badalona, Spain

14 Interventional Radiology, University Hospital of La Ribera, Alcira, Valencia, Spain

15 Interventional Radiology, University Regional Hospital of Málaga, Málaga, Spain

16 Interventional Radiology, San Carlos University Clinical Hospital, Madrid, Spain

17 Interventional Radiology, Parc Tauli Hospital, Sabadell, Spain 
There are many general rules and regulations [9] that are constantly being modified after the COVID-19 outbreak. Despite the fact that there are guidelines and protocols developed urgently by the Spanish Ministry of Health, by the Health Departments of the different Autonomous Communities and by some third-level university hospitals, no one like the IRs will know the singularity of this actual problem in IR departments [10-12]. In order to guarantee patients a high quality of care, specific safety among professionals, a group of experts has prepared this consensus document for the management and infection control of the COVID-19 outbreak for Vascular and Interventional Radiology Departments.

\section{Strategy in IR Departments}

Patient assistance organization, within IR departments, is to create independent staff segregation teams including nurses and physician (in those hospitals where is possible), apply social distancing and interact the least possible with each other. In this way, in the event of an infection or a member being quarantined, there may be other team "clean" and available to perform any daily or emergency procedure. It is highly recommended to distribute the activity in shifts being essential that the teams have different work schedules like alternate days or weeks, in morning or evening schedules, etc. Another recommendation is to treat patients also in schedules and not to mix inpatients with outpatients, and also transform IR inpatients into IR outpatient procedures to minimize infection rates.

Interventional procedures will preferably evaluate as therapeutic alternatives of surgical or endoscopic procedures that might generate aerosols [13, 14], always in accordance with what is the best treatment for the patient, but also prevent healthcare workers transmission.

\section{Indications and Patient Care}

In this global emergency situation, it is crucial to reduce activity and avoid the circulation of patients and healthcare personnel, performing only those treatments without the possibility of a delay. Elective procedures must be postponed (Table 1). It is important to consider that in IR departments, more than $40 \%$ of their activity corresponds to oncological patients. For this reason, in each oncological case, their priority and the possibility of delayed treatment for a limited period of time should be agreed upon in a multidisciplinary way. The patient whose treatment can be delayed will be properly informed of the exceptional circumstances, as well as the risk/benefit of being admitted to the hospital.

\section{Preventive Measures and Regulations}

\section{General Recommendations in the Care of All Patients}

- There must be maximum coordination in the appointment and arrival of patients into the IR department and also clear coordination to transport the patient to the interventional suite. Avoid the coincidence of incoming and outgoing patients, and minimize waiting periods in the preparation room.

- The transfer from the hospitalization room, emergency room or ICU must be done with the patient covered with a surgical mask that will be used throughout the intervention and its subsequent return transfer. The orderlies or porters responsible for moving the patient must also be equipped with protective gear, including mask/respirator, face shield or goggles, and gloves.

- In the preparation room, the patient will be asked about possible respiratory symptoms and his temperature will be measured.

\section{Care for Patients Without Suspicion or Confirmation of COVID-19 Infection}

There is the possibility of having to treat asymptomatic or undiagnosed patients with COVID-19, so preventive and protective measures must be used at all times, especially in patients derived from the emergency department, ICU and elderly patients from nursing homes.

\section{General Recommendations}

- The patient should wear a surgical mask and surgical cap at all times.

- Doctors, nurses and assistants: hand washing, surgical mask, sterile and waterproof gown, sterile gloves, face shield or goggles, and surgical cap.

\section{Care for Patients with Suspected or Confirmed COVID-19 Infection}

\section{General Recommendations}

- Attention exclusively by health personnel is essential for carrying out the procedure.

- Keep the doors of the interventional suite always closed.

- Only the basic material should be kept in the interventional suite, and leave the alternative materials/devices that may be needed in the preparation area (outside the 
Table 1 Interventional radiology procedures in the context of healthcare emergency by COVID-19 outbreak (modified from RSNA)

\begin{tabular}{lc}
\hline Urgent and emergent procedures & Nonurgent procedures with the possibility of a delayed IR treatment \\
\hline $\begin{array}{l}\text { Embolization-related procedures } \\
\begin{array}{l}\text { Massive acute focal bleedings. Iatrogenic, upper or lower } \\
\text { gastrointestinal, traumatic, postpartum, hemoptysis, renal } \\
\text { angiomyolipoma }\end{array}\end{array} \quad \begin{array}{l}\text { Prostate, uterine fibroid, pelvic congestion syndrome, endoleaks in } \\
\text { endovascular aortic repair, pulmonary arteriovenous malformation, } \\
\text { peripheral arteriovenous malformation, chronic lymph pathology, } \\
\text { hemorrhoidal }\end{array}$
\end{tabular}

\section{Diagnostic procedures}

Tumor biopsies*

Drainage procedures

Clinical signs of sepsis or liver or kidney acute obstructive failure. Percutaneous biliary, cholecystostomy, abdominal abscess, pelvic abscess, thoracic abscess, nephrostomy, empyema, paracentesis

\section{Gastrointestinal-related procedures}

Colon, esophageal, gastrointestinal stenting for acute obstruction, biliary stenting acute neoplastic obstruction, percutaneous gastrostomy in patients without the possibility of nasogastric tube feeding in oncological patients

\section{Venous procedures}

Superior vena cava syndrome revascularization, peripherally inserted central catheters, ports, central venous, catheters in oncological patients, angioplasty or thrombectomy of arteriovenous fistula shunt in hemodialysis patients, inferior vena cava filter implantation, catheter-directed therapy (thrombolysis and/or thrombectomy) for massive pulmonary embolism, submassive pulmonary embolism, proximal deep vein thrombosis

\section{Revascularization procedures}

Stroke, critical limb ischemia, superior mesenteric thrombosis or embolism, portal thrombosis

\section{Locoregional tumor treatment $*$}

Radiofrequency, electroporation, microwave ablation for malignant tumors, transarterial chemotherapy embolization and transarterial radioembolization

Special interventional radiology procedures

Transjugular intrahepatic portosystemic shunt and balloon-occluded retrograde transvenous obliteration in upper gastrointestinal bleeding secondary to esophageal varices
Venographies, arteriographies and benign tumor biopsies

Percutaneous procedures without clinical signs of sepsis. Exchange of noninfectious drainage catheters

Percutaneous gastrostomy in patients with the possibility of nasogastric tube feeding, exchange of noninfectious gastrointestinal, biliary or other catheters

Chronic venous disease, lower limb varices, exchange of noninfectious central vein catheters, inferior vena cava retrieval

Noncomplicated endovascular thoracic or abdominal aneurism repair. Lower limbs arterial (Rutherford $\leq$ III), carotid stenting (nonsymptomatic or without critical stenosis)

Benign tumor radiofrequency or microwave ablation

Transjugular intrahepatic portosystemic shunt in refractory ascites

* Locoregional oncological IR treatments, as well as biopsies of tumors, may be delayed until the COVID-19 outbreak is resolved according to multidisciplinary criteria and once the risk/benefit of each particular case has been assessed

interventional suite). An assistant or nurse should be able to supply it to the room if it is needed.

- Use of PPE:

- Patient: equipped with a surgical cap, and a surgical mask, NOT an FFP2/FFP3 mask/respirator.

- Nurse, IR and other assistants inside the interventional suite: hand washing, reinforced and waterproof gown (if it is not possible, use a plastic apron inside), two sets of boot covers or overshoes, double pair of gloves, face shield, goggles or plastic screen, double surgical cap, mask/respirator N95/N99 or FFP2/FFP3 and a surgical mask over respirator and radiation protection aprons (Fig. 1).
- Avoid leaving the interventional suite with contaminated items of the PPE.

- Dispose of all used and unused material and PPE in specially designated containers.

\section{Recommendation for the Mobilization of Patients from Bed to the Interventional Suite Table}

- The interventional radiology suite must be prepared before the patient's transfer.

- Remove nonessential and mobile equipment to avoid contamination. 
SEQUENCE FOR PUTTING ON PERSONAL PROTECTIVE EQUIPMENT (PPE) IN AN INTERVENTIONAL RADIOLOGY ANGIO SUITE DURING COVID- 19 OUTBREAK.
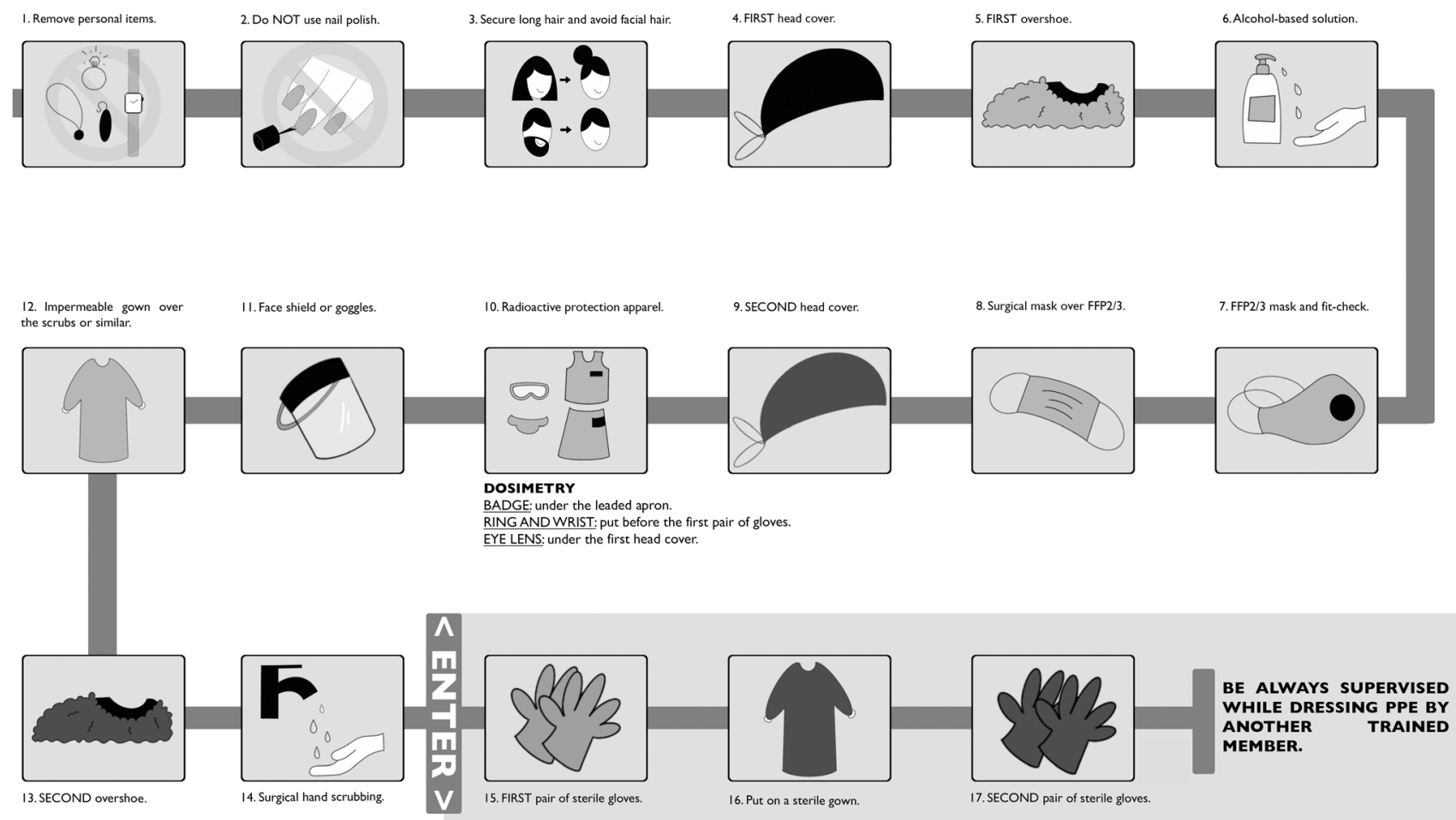

Fig. 1 Sequence for putting on personal protective equipment (PPE) in an interventional radiology interventional suite during the COVID-19 outbreak. FFP: filtering facepiece. ABSHS: alcohol-based solution hand scrub

- Immobile or essential equipment must be covered with plastic covers.

- Donning PPE before the patient arrives.

- The personnel in charge of transferring the patient to the interventional suite must wear a PPE.

- At the end of the patient's transfer, safe removal of the PPE must be performed (Fig. 2).

\section{Recommendation for How to Put on the PPE}

- A schematic diagram in Figure 1 shows step by step proper donning of the PPE.

- It is important to place printed copies in designated areas for personnel to follow step by step.

- In an interventional radiology procedure in which aerosols could be produced, a powered air-purifying respirator (PAPR) should be used in the case of aerosols, if the device is available (Table 2).

\section{Recommendation for How to Remove the PPE}

- A schematic diagram in (Fig. 2) shows step by step proper doffing of the PPE.

- It is important to place printed copies in designated areas, for personnel to follow step by step.

\section{Recommendation After the Completion of the IR Procedure}

- Orderlies or porter will always wear a complete PPE for the patient's transfer.

- The patient will wear a surgical mask AT ALL TIMES.

- All reusable material should be washed and disinfected with wipes or disinfectant liquid (sodium hypochlorite solution), using gloves.

- All the material used in the procedure must be discarded in a special container (biohazard sign for infectious and sharps waste).

- It is recommended take bath and change scrubs after the procedure. 
SEQUENCE FOR REMOVING PERSONAL PROTECTIVE EQUIPMENT (PPE) IN AN INTERVENTIONAL RADIOLOGY ANGIO SUITE DURING COVID- 19 OUTBREAK.

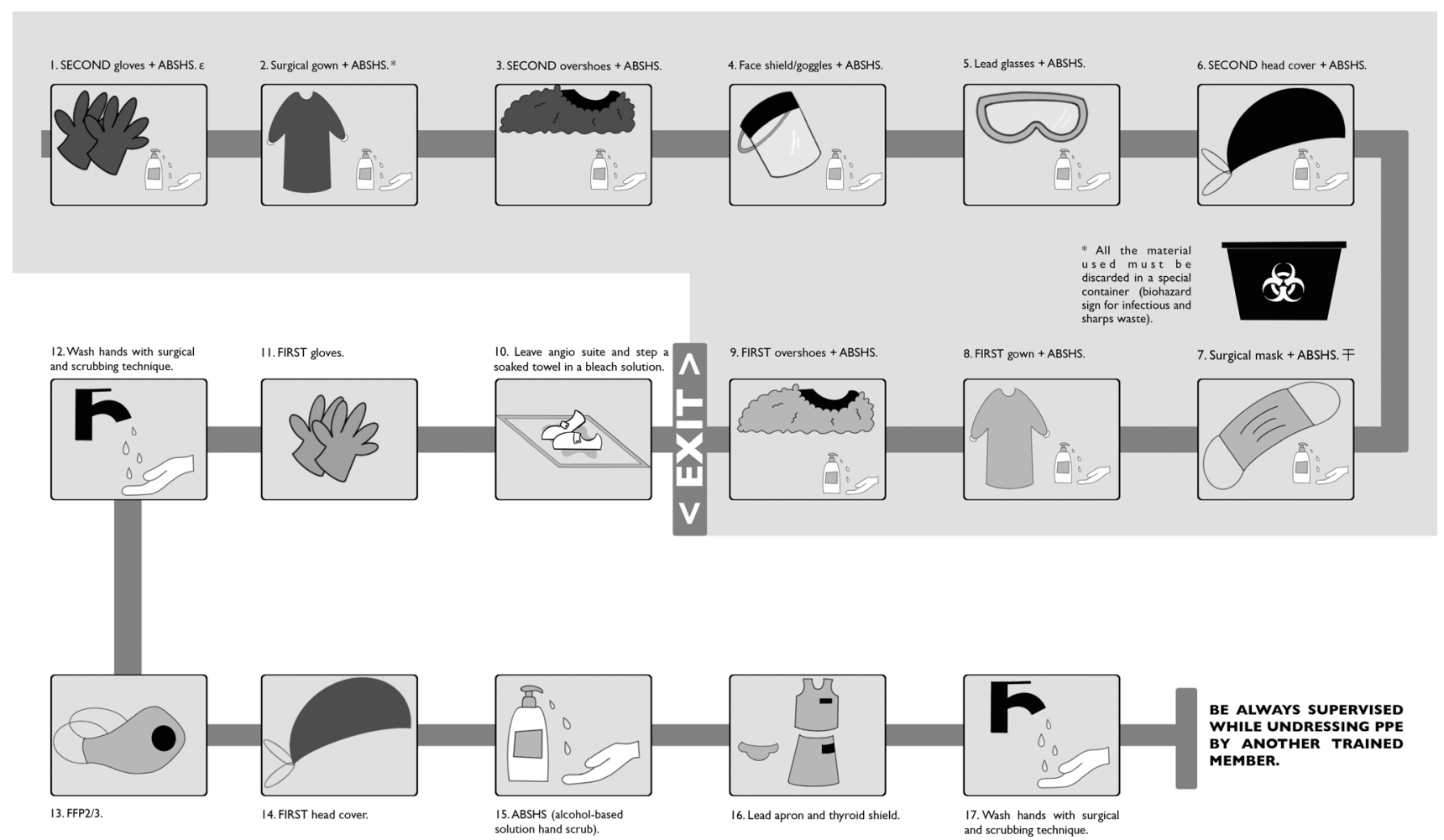

Fig. 2 Sequence for removing personal protective equipment (PPE) in an interventional radiology interventional suite during the COVID19 outbreak. FFP: filtering facepiece. ABSHS: alcohol-based solution hand scrub. The naming sequence of gloves, head covers and overshoes is the same as Fig. 1. $\varepsilon$ : During removal, avoid mobilizing excessively the second pair of gloves and surgical gown to prevent the production of aerosols. ₹: surgical mask, gloves and gown; remember that the outside is contaminated. If your hands get contaminated during removal, immediately wash your hands or use an alcohol-

\section{Recommendations for Cleaning the Interventional Suite}

- Cleaning staff MUST ALWAYS be protected with PPE.

- The interventional suite will be cleaned according to a specific contact and drop isolation procedures with bleach solutions.

- All spaces that may have come into contact with the patient will be cleaned.

- It is recommended that cleaning be carried out 30-60 min after the procedure (aerosol deposit).

\section{Important}

1. Surgical gowns or gloves MUST NEVER LEAVE, the interventional suite. based hand sanitizer. To remove face shield/goggles, start from the back by lifting the headband. To remove gowns, unfasten ties, start from the neck tie and take care also that the sleeves do not touch your body. Turn the gown inside out, and fold it. To remove surgical or FFP2/3 mask/respirator, grasp the bottom elastics of the mask and move with the fingers at the top and remove it without touching the front. If items are not reusable, discard them in a specific waste container (biohazard sign for infectious and sharps waste). If the item is reusable, place it in a designated area for proper sterilization

2. You cannot stay or enter in the interventional suite without wearing the FPP2-3 mask/respirator when a COVID-19 patient is being treated.

3. Wash your hands as many times as necessary with an alcohol-based solution or hydroalcoholic gel.

4. There is evidence of drug interactions with experimental COVID-19 therapies. In any case, consult Anesthesia, Intensive Care or Internal Medicine physicians before administering any drug. For drug interactions, refer https://www.covid19-druginteractions.org/ [15].

\section{Special Situations and Concerns in IR Departments}

IRs also routinely perform procedures guided by computed tomography and ultrasound. Contamination of this equipment constitutes an additional problem for all professionals 
Table 2 Aerosols-generating procedures in the setting of healthcare emergency by COVID-19 outbreak (modified table of CIRSE-APSCVIR checklist)

Aerosol-generating procedures (AGPS)

Cardiac- and pulmonary-related procedures

Intubation and extubation, manual ventilation, positive-pressure ventilation, nebulizer treatment, high-flow oxygenation nasal cannula therapy, chest physiotherapy, bronchoscopy, cardiopulmonary resuscitation (CPR)

Interventional radiology procedures

Procedures or patient having or generating cough or hemoptysis: hemoptysis embolization, nasogastric tube placement, esophageal stent placement, lung biopsy, pleural drainage, lung ablation, bronchial artery embolization, pulmonary embolism with catheter-directed therapy either thrombolysis or thrombectomy.

Suctioning procedures

Airway, oral and tracheostomy.

Oral, nasal and dental procedures

Drilling in dental procedures, nasopharyngeal/oral pharyngeal swab collection, sputum induction, or other nasal or oral procedures

Gastrointestinal-related procedures

Gastroesophageal endoscopy, nasogastric tube insertion

Any other procedures via the nasal or oral routes

Surgical procedures

Tracheostomy creation/insertion, high-speed devices used for surgical procedures, laparoscopic or laparotomy surgical procedures

Powered air-purifying respirator (PAPR) is recommended when performing AGPS on suspected or confirmed COVID-19 patients, nonetheless if shortage or nonavailable PAPR whole face protection is needed (face shield, goggles, FFP2/3 mask and waterproof hood)

who perform these procedures. Decontamination and sterilization of CT rooms and interventional ultrasound rooms are a major difficulty and concern. On the other hand, the IR departments have: computers, screens, keyboards, remote controls, microphones, anesthesia monitors and mechanical ventilators, monitoring devices and other types of electronic or office equipment that will require protection and special attention to avoid fomite transmission.

In hospitals where sufficient technology is available, one of them could be selectively dedicated to COVID-19 patients, reserving the other equipment for unsuspected and non-COVID-19 patients. Interventional radiology suites are recommended to prepare and reserve at least one interventional suite for COVID-19 patients [16]. It is also important to minimize moving COVID-19 patients and determine whether it is possible to perform procedures with portable bedside ultrasound, always in accordance with what is the best care of the patient, and to evaluate the risk of complications working outside of the IR environment [12].

\section{Suggestions for the Organization of Patient Care Activity in an IR Department (Variable According to Epidemiological Scenario and Availability of Each Hospital)}

The American Society for Interventional Radiology (SIR) on its Web site [17] recommends assessing the risk of COVID-19 infection (low, medium and high) based on the geographical area and within it according to the healthcare setting (outpatients or hospitalized patients).

In high-risk areas, it is recommended to cancel all procedures except urgent or emergent ones, as other colleagues have already decided [18]. However, it also recommends individualizing each case based on the epidemiological scenario and the possibilities of the hospital center (human and physical resources) (Table 1).

\section{Conclusion}

The most important recommendations for the Vascular and Interventional Radiology Departments in the COVID-19 outbreak are: (1) create separate teams of IRs and nurses (two or three groups according to the availability) to increase the guarantee of care and to reduce casualties; (2) to carry out a checklist of all the patients who come to the interventional suite, classify them into positive COVID-19, uncertain or negative and use the appropriate protective measures; (3) all patients (infected and noninfected) who arrive at the interventional suite must be provided with a surgical cap and mask; (4) all the health workers who receive a COVID-19 patient in the interventional suite must also be protected with a complete PPE; and (5) IR activity must be reduced only to specific cases in which the procedure is necessary by all means or cannot be delayed.

The data that appear in this document are from the current scenario with the information updated on April 7, 2020. This consensus document was issued for Spain with 
the particular phase of the outbreak, and the institutions will need to adapt it based on their respective institutional guideline and phase of the COVID-19 outbreak.

Acknowledgements The authors thank Dr. Lucía M. Marroquín and Dr. Rodolfo A. Estrada for the English language editing of this manuscript.

Funding Authors declare there was no funding.

\section{Compliance with Ethical Standards}

Conflict of interest Authors declare they have no conflict of interests.

Consent for Publication Consent for publication was obtained for every individual person's data included in the study.

Ethical Approval For this type of study, ethical approval for publication was not necessary.

Informed Consent For this type of study, consent for publication is not required.

\section{References}

1. Coronavirus disease (COVID-19). Pandemic [Internet]. [cited 2020 April 7]. Available from: https://www.who.int/emergencies/ diseases/novel-coronavirus-2019.

2. Guan W-J, Ni Z-Y, Hu Y, Liang W-H, Ou C-Q, He J-X, et al. Clinical characteristics of coronavirus disease 2019 in China. N Engl J Med. 2020.

3. Real Decreto 463/2020, de 14 de marzo, por el que se declara el estado de alarma para la gestión de la situación de crisis sanitaria ocasionada por el COVID-19. [Internet]. 2020 [cited 2020 Mar 28]. Available from: https://www.boe.es/eli/es/rd/2020/03/14/ 463.

4. Coronavirus Update (Live): 1,410,150 Cases and 81,012 Deaths from COVID-19 Outbreak-Worldometer [Internet]. [cited 2020 April 7]. Available from: https://www.worldometers.info/ coronavirus/.

5. Romaguera R, Cruz-González I, Ojeda S, Jiménez-Candil J, Calvo D, García Seara J, et al. Consensus document of the Interventional Cardiology and Heart Rhythm Associations of the Spanish Society of Cardiology on the management of invasive cardiac procedure rooms during the COVID-19 coronavirus outbreak. REC Interv Cardiol [Internet]. doi:https://doi.org/10. 24875/RECIC.M20000114.

6. Coronavirus: España tiene 9.444 sanitarios infectados, un $75 \%$ más en 3 días [Internet]. Red Médica. [cited 2020 Apr 07]. Available from: https://www.redaccionmedica.com/secciones/ sanidad-hoy/coronavirus-espana-tiene-9-444-sanitariosinfectados-un-75-mas-en-3-dias-7049.

7. Urbano J, De Gregorio MA. Plan Estratégico de SERVEI I Sociedad Española de Radiología Vascular e Intervencionista
(SERVEI) [Internet]. [cited 2020 Mar 28]. Available from: https://servei.org/plan-estrategico-servei/. https://doi.org/10. 30454/978-84-09-07070-1.

8. Urbano J, de Gregorio MA. The strategic plan of Spanish Society of vascular and interventional radiology. Cardiovasc Intervent Radiol. 2019;42:632-5.

9. Procedimiento de actuación frente a casos de infección por el nuevo coronavirus (SARS-CoV-2) [Internet]. 2020 [cited 2020 Mar 28]. Available from: https://www.mscbs.gob.es/ profesionales/saludPublica/ccayes/alertasActual/nCov-China/ documentos/Procedimiento_COVID_19.pdf.

10. Chandy PE, Nasir MU, Srinivasan S, Klass D, Nicolaou S, Babu SB. Interventional radiology and COVID-19: evidence-based measures to limit transmission. Diagn Interv Radiol Ank Turk. 2020. Published online 27 March 2020. https://doi.org/10.5152/ dir.2020.20166.

11. Lau T-N, Teo N, Tay K-H, Chan L-L, Wong D, Lim WEH, et al. Is your interventional radiology service ready for SARS?: The Singapore experience. Cardiovasc Intervent Radiol. 2003;26:421-7.

12. Da Zhuang K, Tan BS, Tan BH, Too CW, Tay KH. Old threat, new enemy: is your interventional radiology service ready for the coronavirus disease 2019? Cardiovasc Intervent Radiol. [Internet]. 2020 [cited 2020 Apr 6]; Available from: https://doi.org/ 10.1007/s00270-020-02440-6.

13. Tran K, Cimon K, Severn M, Pessoa-Silva CL, Conly J. Aerosol generating procedures and risk of transmission of acute respiratory infections to healthcare workers: a systematic review. PLoS ONE. 2012;7:e35797.

14. COVID-19 resource centre [Internet]. CIRSE. [cited 2020 Apr 6]. Available from: https://www.cirse.org/wp content/uploads/2020/ 04/CIRSE_APSCVIR_COVID19_Checklist.pdf.

15. Detailed recommendations for interactions with experimental COVID-19 therapies. [Internet]. COVID-19 Drug Interact. 2020 [cited 2020 Mar 29]. Available from: https://www.covid19druginteractions.org/https://www.covid19-druginteractions.org/ rails/active_storage/blobs/eyJfcmFpbHMiOnsibWVzc2FnZSI6IkJBaHBDdz09IiwiZXhwIjpudWxsLCJwdXIiOiJibG9iX2lkIn19-90a290196d33633bf481bab8c06509bb73e10cc3/ Covid_InteractionDetails_Web_2020_Mar20.pdf.

16. Tsou IYY, Goh JSK, Kaw GJL, Chee TSG. Severe acute respiratory syndrome: management and reconfiguration of a radiology department in an infectious disease situation. Radiology [Internet]. Radiological Society of North America; 2003 [cited 2020 Apr 5]; Available from: https://pubs.rsna.org/doi/abs/10.1148/ radiol.2291030789.

17. Society of Interventional Radiology-SIR COVID-19 Resources for IR [Internet]. [cited 2020 Mar 29]. Available from: https:// www.sirweb.org/practice-resources/covid-19-resources/.

18. COVID-19 Guidelines for Triage of Emergency General Surgery Patients [Internet]. Am Coll Surg. [cited 2020 Mar 28]. Available from: https://www.facs.org/covid-19/clinical-guidance/electivecase/emergency-surgery.

Publisher's Note Springer Nature remains neutral with regard to jurisdictional claims in published maps and institutional affiliations. 